\title{
Sintering, camber development of layer composites and a new method to eliminate or decrease camber
}

\author{
XIANGCHUN LIU \\ Department of Materials Science and Engineering, Xi' an University of Science and Technology, Xi' an 710054, P. R. China
}

MS received 17 February 2012; revised 3 December 2012

\begin{abstract}
Zinc-magnesium titanate dielectrics/nickel-zinc-copper ferrite layer composites were prepared by tapecasting technique combined with a uniaxial pressure shaping technique. The sintering and camber development of the composites were investigated. The results show that the difference of shrinkage in the later stages of sintering is the main cause of camber distortion. To eliminate or decrease the camber, a new method semi-fixed uniaxial pressure technique (SUP) was proposed. This method uses uniaxial pressures by which different radial densities can be introduced when one layer's edge is clamped and the other is free while laminating two different layers. The results obviously show that the SUP technique can decrease the camber and the camber curvature shows an inverse quadratic dependence on the applied pressure.
\end{abstract}

Keywords. Tape-casting; layer composites; camber curvature; co-sintering.

\section{Introduction}

Multilayer dielectric-magnetic composites exhibiting both inductive and capacitive properties are a solution to the fabrication of miniature filters and antennas, control of electromagnetic interference (EMI) (Merja et al 2010). They are made by stacking ferrite layers and dielectric layers with internal electrode pastes, and then co-fired. The sintering temperature of ferrite and dielectrics must be below $900{ }^{\circ} \mathrm{C}$ to ensure their cofiring with Ag internal electrode. The properties of devices depend on the properties of the sintered ferrites, dielectrics and quality of the interface. On the one hand, interfacial diffusion is inevitable, and this process can affect properties near the interface. On the other hand, the mismatch in sintering kinetics between different compositions of ceramics may cause residual stresses or cracks. Therefore, the matching and compatibility during lowtemperature co-firing process is the key to achieving multilayer chip composites.

The sintering temperature of hexagonal ilmenite-type $\left(\mathrm{Zn}_{0.7} \mathrm{Mg}_{0.3}\right) \mathrm{TiO}_{3}$ (abbreviated as ZMT3) dielectric ceramics was lowered from $1200{ }^{\circ} \mathrm{C}$ to $875^{\circ} \mathrm{C}$ successfully by a semichemical route and $1.0 \mathrm{wt} \% \mathrm{~V}_{2} \mathrm{O}_{5}$ additions which provided a significant benefit to develop $\mathrm{LTCC} \mathrm{ZnTiO}_{3}$-based ceramics (Liu et al 2007; Zhou et al 2008; Jiao et al 2010). $\mathrm{NiCuZn}$ ferrite, which has excellent magnetic permeability and can be sintered at low temperature, is an important

liuxc@126.com material used for producing low temperature sintered multilayer chip inductor (MLCI) (Jean and Lee 1999; Seo and Oh 1999). They are promising materials for the multilayer chip LC filters.

In our previous work, zero camber and crack-free $\mathrm{ZnTiO}_{3}$ dielectrics/NiZnCu hexagonal ferrite (abbreviated as ZT/NZC) composite samples were prepared successfully by using restricted shrinkage sintering process (RSS) (Liu et al 2009a, b). But the electromagnetic performance degradation of co-sintered layer composition samples was obvious because of the decomposition of $\mathrm{ZnTiO}_{3}$. To solve this problem, ZMT3/NZC/ZMT3 and NZC/ZMT3/NZC sandwich layer composites were designed and prepared by a novel zero-shrinkage-difference (ZSD) technology combined with controlling cooling stage of the sintering process (Liu et al 2009a, b). But in some NZC/ZMT3/NZC samples, cracks in interface and channel cracks in ZMT3 layer were observed. These results show that the difference in radial shrinkage induces internal constraints and interfacial stresses. So, it is important to understand the temperature stresses generation, sintering kinetics and camber development in layer composition and to control their radial shrinkage (Green et al 2008). Essentially, the internal stresses, which have the potential to hinder densification or lead to defects and/or distortions in the fired bodies, usually result from mismatches in the radial shrinkage behaviour of the various components (Chang et al 2007). So, in the present study, a simpler layer composition, the bilayer composite, were prepared by cofiring ZMT3 dielectrics and NiCuZn (abbreviated as NZC) ferrite at $900{ }^{\circ} \mathrm{C}$ in order to investigate the sintering kinetics and camber development of co-fired composites. 


\section{Experimental}

NZC ferrite and ZMT3 powders were synthesized by solidstate reaction method combined with a chemical processing. $\left(\mathrm{Zn}_{1-x} \mathrm{Mg}_{x}\right) \mathrm{TiO}_{3}(x=0$ to $0 \cdot 5)$ system was prepared using a semichemical route with A.R. zinc hydroxide carbonate $\left(\mathrm{Zn}_{5}\left(\mathrm{CO}_{3}\right)_{2} \cdot(\mathrm{OH})_{6}\right.$, Beijing Chemical Reagent Company), A.R. magnesium hydroxide carbonate $\left(\mathrm{Mg}(\mathrm{OH})_{2}\right.$. $4 \mathrm{MgCO}_{3} \cdot 6 \mathrm{H}_{2} \mathrm{O}$, Tianjin Chemical Reagent Company) and anatase $\left(\mathrm{TiO}_{2}, 10 \sim 30 \mathrm{~nm}, \mathrm{Xi}\right.$ ' an Chemical Reagent Company). The carbonates were heat-treated at 350 and $540{ }^{\circ} \mathrm{C}$ for $2 \mathrm{~h}$ in air to obtain nanopowder $\mathrm{ZnO}(10 \sim 30 \mathrm{~nm})$ and $\mathrm{MgO}(20 \sim 50 \mathrm{~nm})$ with high active energy as starting materials, respectively. X-ray diffractometry (XRD) confirmed that pure phase $\mathrm{ZnO}$ and $\mathrm{MgO}$ were obtained. Anatase nanopowder was mixed with $\mathrm{ZnO}, \mathrm{MgO}$ and dispersant, according to the composition of $\left(\mathrm{Zn}_{0.7} \mathrm{Mg}_{0.3}\right) \mathrm{TiO}_{3}$, using planetary milling with zirconia balls in ethanol for $24 \mathrm{~h}$. The mixture was dried and then calcined at $750{ }^{\circ} \mathrm{C}$ for $2 \mathrm{~h}$. The resultant powders were the ZMT3 starting material.

Next, 99\% pure reagent-grade $\mathrm{NiO}$ (Xi'an Chemical Reagent Company), $\mathrm{Fe}_{2} \mathrm{O}_{3}$ (Xi' an Chemical Reagent Company), $\mathrm{CuO}$ (Xi'an Chemical Reagent Company) and high active $\mathrm{ZnO}$ nanopowder, according to the composition of $\left(\mathrm{Ni}_{0.8} \mathrm{Cu}_{0.12} \mathrm{Zn}_{0.12}\right) \mathrm{Fe}_{1.96} \mathrm{O}_{4}$, were weighed and mixed for $12 \mathrm{~h}$ in a planetary ball mill. The mixtures were calcined $\left(720^{\circ} \mathrm{C}\right.$ for $\left.4 \mathrm{~h}\right)$ as NZC starting material.

Then, the sintering additives $\left(\mathrm{V}_{2} \mathrm{O}_{5}\right.$ for $\mathrm{ZMT} 3, \mathrm{Bi}_{2} \mathrm{O}_{3}$ for $\mathrm{NZC}$ ), solvent, dispersant, binder, and plasticizer were mixed into the ZMT3 starting material and NZC starting material, respectively. The two mixtures were milled for $48 \mathrm{~h}$ each to prepare the slurries.

After sifting out and removing the air bubbles, the slurries were tape-casted into green sheets of $100 \mu \mathrm{m}$ in thickness. Then, the green sheet was cut into a round sheet with a diameter of $12 \mathrm{~mm}$ that is equal to the diameter of the mould which was used to introduce a uniaxial pressure. 15 20
NZC round green sheets were laminated into a thick sheet of about 1.5 2 mm in height; and the ZMT3 thick green sheet (1.5 2 $\mathrm{mm}$ high) was prepared by the same procedure. The lamination condition was $20 \mathrm{MPa}$ at $60{ }^{\circ} \mathrm{C}$ for $10 \mathrm{~min}$. The layer samples were assembled by stacking NZC round thick sheet on the top side of the ZMT3 round thick sheet. Then, the layer samples were pressed using a uniaxial pressure of 2 8 MPa with the NZC's edge being clamped and the ZMT3's edge being left free. After the uniaxial pressure, diameter of ZMT3 was bigger than that of NZC. So, the excess edge had to be cut off. The final dimensions of the laminated green samples were $12 \times 1.5 \mathrm{~mm}$ and $12 \times 2 \mathrm{~mm}$. The procedures for preparing round sheet and layer samples are shown in figure 1. (The reasons for clamping one layer's edge and leaving the other free are explained in detail later). The binder was burnt out by heating up very slowly to $350{ }^{\circ} \mathrm{C}$, and kept for $6 \mathrm{~h}$ at this temperature. The samples were sintered at $900{ }^{\circ} \mathrm{C}$ in air for $4 \mathrm{~h}$.

The sintered densities were measured by the Archimedes method. The macrograph photograph was captured by metallurgical microscope (OLYMPUS PMG3).

\section{Results and discussion}

The camber is caused mainly by the difference in shrinkage in co-sintering two different layers. To research the sintering shrinkage of ZMT3 and NTC ceramics, before the lamination samples were prepared and co-sintered, single ZMT3 and NTC ceramics were prepared by solid-state reaction using the same starting material as the lamination samples. The radial shrinkage of single annealing sample was measured by vernier caliper.

The radial shrinkage curves of ZMT3 and NZC are shown in figure 2. (the experimental values are geometric averages of five samples). It can be seen that the shrinkage of both samples increased as the sintering temperature increased

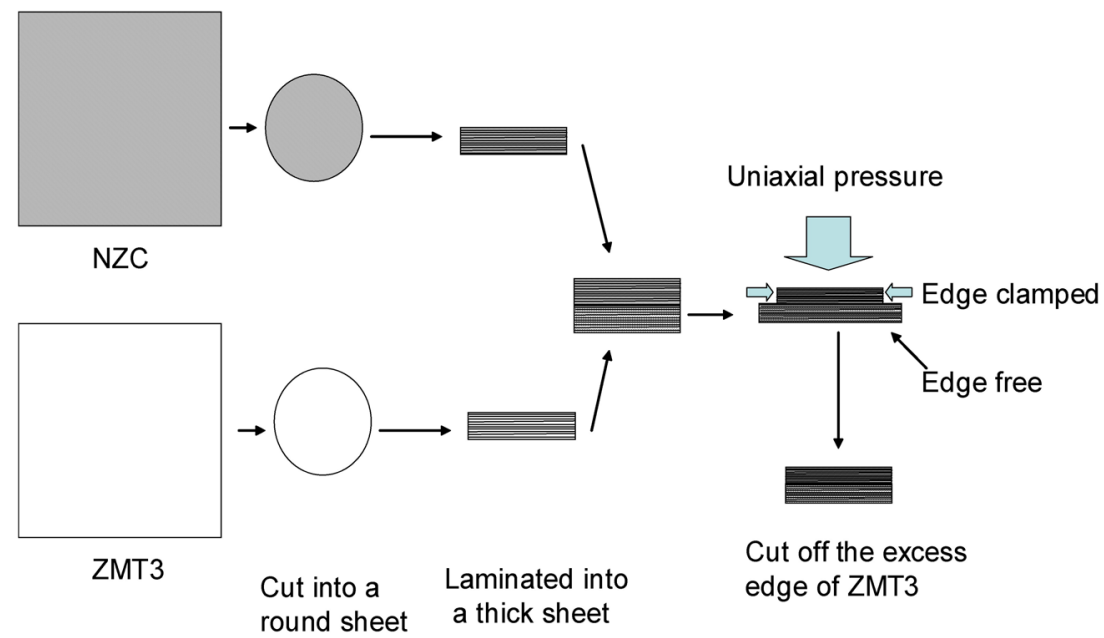

Figure 1. Procedure of preparing multi-layer green sample. 


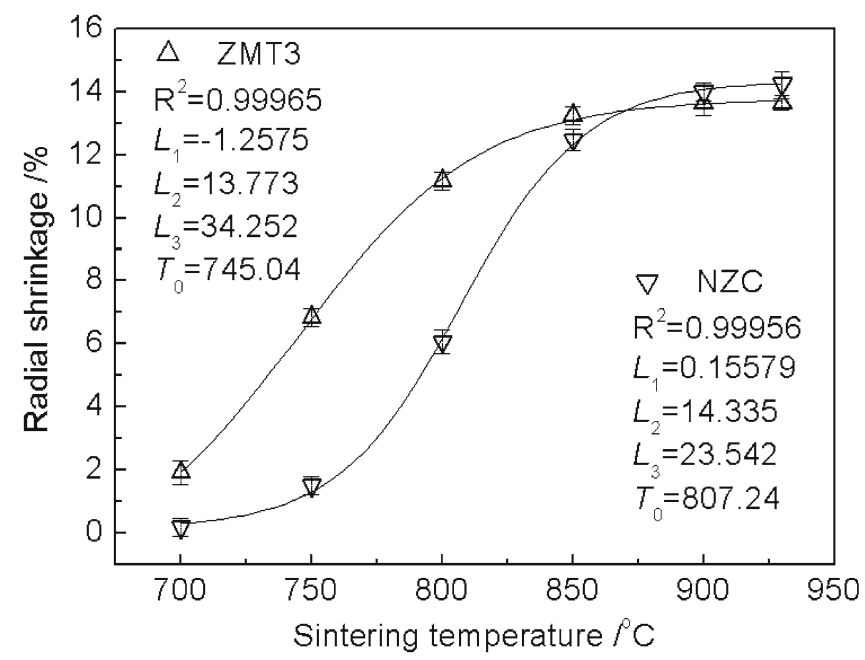

Figure 2. Radial shrinkage curve of ZMT3 and NZC vs sintering temperature.

from 700 to $950{ }^{\circ} \mathrm{C}$. Obviously, the shrinkage of NZC was less than that of ZMT3 at a temperature $<869{ }^{\circ} \mathrm{C}$. When the temperature increased, the shrinkage of NZC became higher than that of ZMT3. At $869{ }^{\circ} \mathrm{C}$, they had the same shrinkage. It is suggested that deformation may not exist when the two materials were co-sintered at $869^{\circ} \mathrm{C}$. However, this is not realistic, because their optimal sintering temperature is at $900 \sim 950{ }^{\circ} \mathrm{C}$. We do not know what effect the difference of shrinkage above and below $869^{\circ} \mathrm{C}$ and the difference of shrinking rate have on the camber development. The difference of shrinking rate can be understood by studying the change curve of shrinkage derivative versus temperature. For nonlinear fitting of the experimental data, a shrinkage equation can be written as

$$
\Delta L / L_{0}=L_{2}+\frac{\left(L_{2}-L_{1}\right)}{1+\exp \left(\left(T-T_{0}\right) / L_{3}\right)},
$$

where $\Delta L / L_{0}$ are defined as sample radial shrinkage. $L_{1}, L_{2}$, $L_{3}$ and $L_{0}$ are constants (their values are shown in figure 2). The correlation coefficients $(R)$ of fitting curves are shown in figure 2 .

Using (1), the derivative of shrinkage with temperature, $d(L / \Delta L) / d T$ can be calculated as

$$
\frac{d(L / \Delta L)}{d T}=\frac{\left(L_{2}-L_{1}\right)}{L_{3}} \frac{\exp \left(\left(T-T_{0}\right) / L_{3}\right)}{\left(1+\exp \left(\left(T-T_{0}\right) / L_{3}\right)\right)^{2}} .
$$

The derivative curve of ZMT3 and NZC is shown in figure 3. It can be seen that the maximum shrinkage of ZMT3 and NZC occurred at 750 and $800{ }^{\circ} \mathrm{C}$, respectively.

According to the above results, in the initial sinter stage (sintering temperature $<869^{\circ} \mathrm{C}$ ), the samples would produce deformations and bend to ZMT3 side because of ZMT3's bigger radial shrinkage. When the samples were sintered at a sintering temperature of $>869^{\circ} \mathrm{C}$, they would produce camber distortion and bend to the NZC side because of NZC's bigger radial shrinkage, as shown in figure 4 (laminating condition was $20 \mathrm{MPa}$ at $60{ }^{\circ} \mathrm{C}$ for $10 \mathrm{~min}$ ). The difference of the

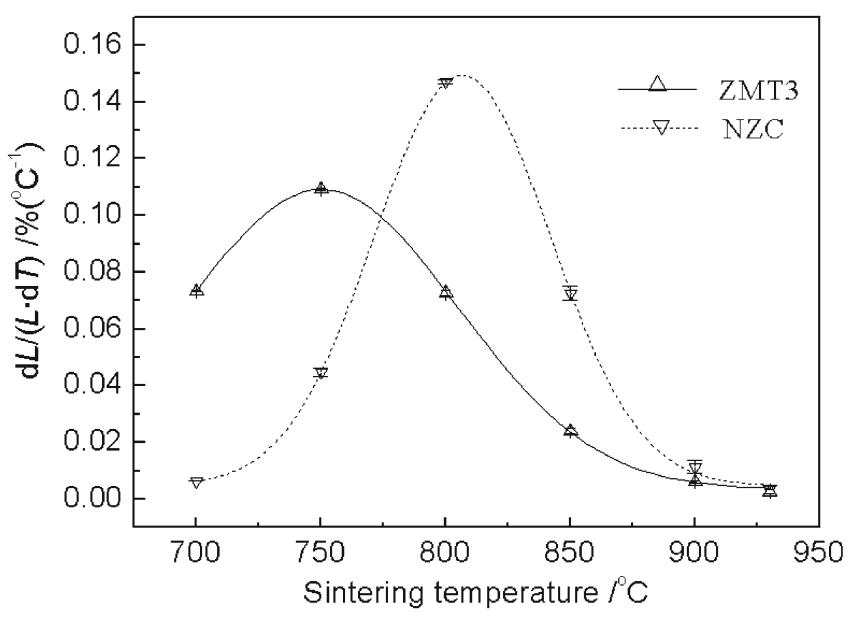

Figure 3. Derivative curve of shrinkage with temperature of ZMT3 and NZC.

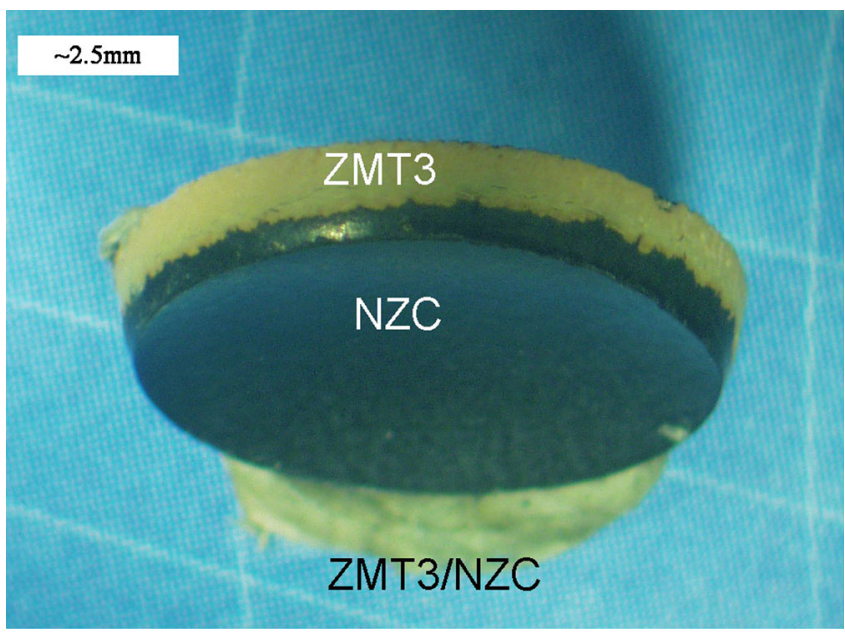

Figure 4. Macrograph of ZMT3/NZC co-fired sample.

derivative of shrinkage with temperature may induce cracks in interface. But the experimental results show that the specimen bend to the NZC side and no cracks were observed, as shown in figure 4 . So, it can be deduced that the difference of shrinkage in the later stages of sintering was the major cause of camber distortion and the difference of shrinking rate would not affect on camber development.

The mechanism of camber can be explained further, according to the structure model of two-layer composite under different conditions, as shown in figure 5 .

For the non-binding free shrinkage of bilayer composite, there was no stress produced between the two layers during sintering. The bodies show volume contraction, as shown in figure 5c. But interfacial reaction between the two layers inevitably led to interfacial stress because of the difference of shrinkage between the two layers, as shown in figure $5 \mathrm{~d}$. In this case, the dielectric layer would densify at $<869{ }^{\circ} \mathrm{C}$, but it was constrained by the non-sintering green ferrite layer. At higher temperatures of $>869^{\circ} \mathrm{C}$, the ferrite 


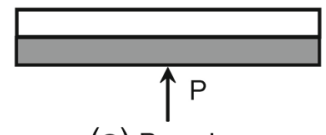

(a) Pressing

(d) Constrained shrinkage

(+, In-plane tension stress;

-, In-plane compression stress)

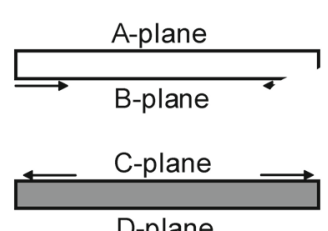

D-plane

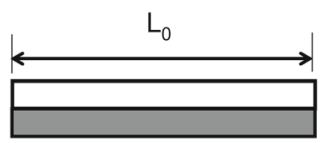

(b) Green sheet

(b) Green sheet

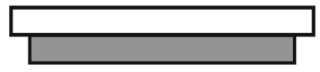

(c) Freedom shrinkage (e) Distortion induced

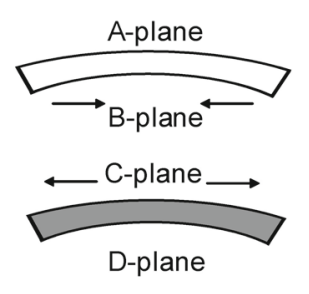

by internal stress

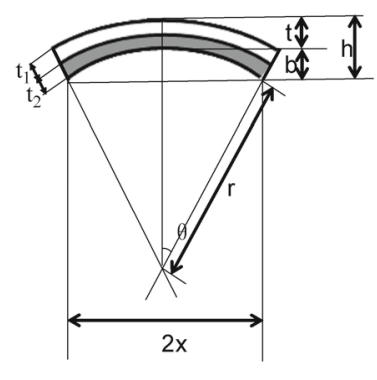

(f) Sintered sample

Figure 5. Structure model of two-layer composite under different conditions.

layer densified, but the shrinkage was inhibited by the sintered dielectric layer. Thereby, a self-constrained sintering is accomplished, because each layer acts as a constraining layer (Cai et al 1997a, b; Hagymasi et al 2005). In the interface of ZMT3/NZC layer composition, an in-B-plane compression stress would be induced because the shrinkage of ZMT3 is less than that of NZC. At the same time, an in-C-plane tension stress was also induced because of the higher shrinkage of NZC (as shown in figures $5 \mathrm{~d}$ and e). As a result, the Bplane radial shrinkage became bigger than the A-plane with zero stress because of the interface stress in B-plane. Similarly, the C-plane radial shrinkage became less than the Dplane with zero stress because of the interface stress in Cplane. At last, the specimen bent to the side with a higher shrinkage (the side of NZC in this work) because the difference of shrinkage in the later sintering stages was the main cause of camber distortion, as shown in figure 5f. The isotropic constitutive models have been proved inadequate for constrained sintering (Bordia et al 2006). Because the key assumption for the validity of the isotropic model is that the microstructure for the constrained layer sample is identical to the microstructure at the same density for a freely sintering sample. In particular, the samples must have the same grain and pore size, shape and orientation (Bordia et al 2006). But, under uniaxial stress, the pores have a nonspherical geometry (like an ellipsoid) with the orientation. Experimental results show that the axial shrinkage was only about $1 \%$. That was much smaller than the radial shrinkage.

According to figure 1 and above results, we classify the process of camber development into three stages. First, the camber goes toward ZMT3 at the temperature below $869^{\circ} \mathrm{C}$. Then, the camber disappears at $869{ }^{\circ} \mathrm{C}$. Finally, it goes toward NZC at the temperature higher than $869{ }^{\circ} \mathrm{C}$. The camber of such a non-external-binding free-shrinkage bilayer composite system can be characterized by the model reported
(Gao et al 2003). The equation used to describe the camber is given as

$$
C=\frac{r(1-\cos \theta)}{2(R+t) \sin \theta},
$$

where $t$ is total thickness of sintered composite, $r$ the camber radius, $\theta$ the camber angle and $C$ represents camber. The meaning of these parameters is explained in figure $5 \mathrm{e}$. However, it is difficult to measure the camber angle from the sintered composites. In this work, no obvious chemical reactions occurred between ZMT3 and NCZ materials (Liu 2012). So, it is assumed that each layer sustains its own shrinkage during the sintering process. The following equations illustrated by figure $5 \mathrm{e}$ are proposed, based on (3), and applied in measuring camber as a simpler and more effective way. So, $\cos \theta, \sin \theta$ and $r$ can be given as

$$
\begin{aligned}
& \cos \theta=\frac{r-b}{r}, \sin \theta=\frac{x}{r}, \\
& r^{2}=x^{2}+(r-b)^{2},
\end{aligned}
$$

where $b$ and $x$ are defined as camber axial distance and camber radial distance, respectively. Then, the camber and curvature can be calculated by (4) and (5) as follows

$$
\begin{aligned}
& C=\frac{\left(x^{2}+b^{2}\right) b}{2 x\left(x^{2}+b^{2}+2 b t\right)}, \\
& \rho=1 / r=\frac{2 b}{x^{2}+b^{2}}
\end{aligned}
$$

where $b, x$ and $t$ can be easily measured by optical microscope and $C$ and $\rho$ can be calculated by (6) and (7), expediently.

The shrinkage difference, the calculated camber and the camber curvature from the experimental data are shown in 
table 1 , where the positive direction of camber is defined as the camber towards NZC. It can be seen that more the shrinkage difference is, the larger the camber and the smaller the camber radius.

From the above analysis, we can conclude that the camber generation and camber curvature were related to the difference of shrinkage of two materials. We can deduce that the camber can be eliminated when two materials have the same shrinkage in the later stages of sintering. As we know, the temperature and pressure can change the stress states and shrinkage during sintering, which are the important factors influencing camber distortion. But it is difficult to eliminate the camber by only changing the co-sintering temperature. So, we attempt to change the radial density and shrinkage of two layers by changing pressures. It is almost impossible to change the radial density and shrinkage of two layers at the same time by changing pressures for the conventional methods.

To obtain different radial densities, a new method viz. 'semi-fixed uniaxial pressure technique', abbreviated as SUP, was proposed, as shown in figure 1 . This method uses uniaxial pressures by which different radial densities can be introduced when one layer's edge is clamped and the other is free, while laminating two different layers.

When the uniaxial pressure along the vertical direction is introduced to NZC layer with edge clamped, the particles tend to fill in the pores along the horizontal plane direction because of the high friction between the particles and between the inner wall of the mould and particles. The residual pores then were distributed along the vertical-plane direction and NZC layer had higher in-plane density and lower perpendicular-plane density. For the ZMT3 layer with free edge, the uniaxial pressures push the particles to fill in the pores in the vertical-plane direction because of the high friction between particles and free edge. Then, the residual pores were distributed along plane direction and the ZMT3 layer had higher perpendicular-plane density and lower in-plane density.

As we know, the smaller the density, the bigger the shrinkage for the same materials. ZMT3 has smaller in-plane density and higher radial shrinkage, which is expected to compensate for the difference of shrinkage between ZMT3 and NZC.

To determine the uniaxial pressure range, the uniaxial pressure dependence of the radial shrinkage for single ZMT3 and NZC ceramics was studied in detail. The results are shown in figure 6 (The experimental values were

Table 1. Camber development with sintering temperature and shrinkage difference of ZMT3 and NZC.

\begin{tabular}{lccc}
\hline $\begin{array}{l}\text { Sintering } \\
\text { temperature }\left({ }^{\circ} \mathrm{C}\right)\end{array}$ & $\begin{array}{c}\text { Shrinkage } \\
\text { difference }(\%)\end{array}$ & $\rho\left(\mathrm{mm}^{-1}\right)$ & $C(\%)$ \\
\hline 875 & 1.08 & 0.02893 & 3.64192 \\
900 & 2.75 & 0.03669 & 4.63883 \\
930 & 4.69 & 0.04456 & 5.49262 \\
\hline
\end{tabular}

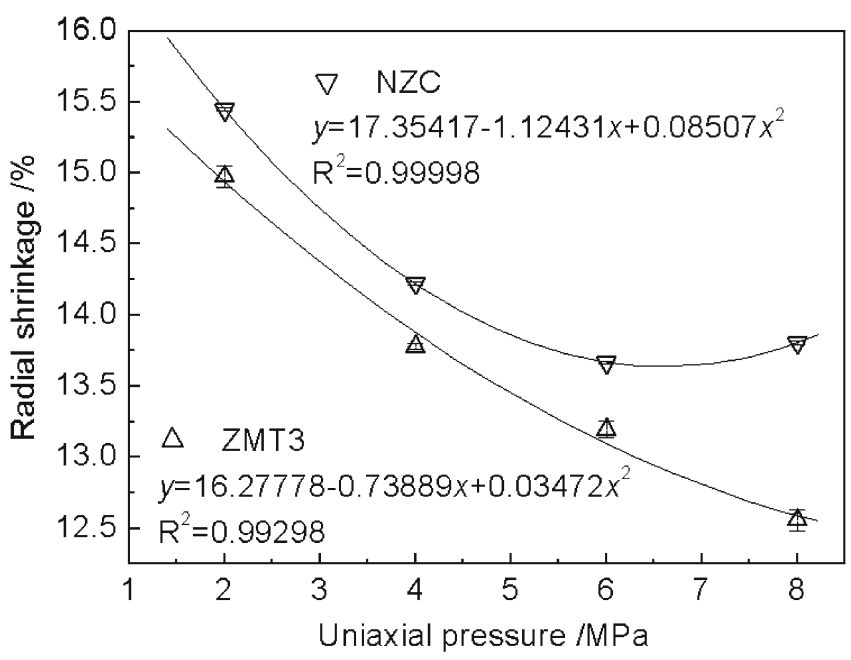

Figure 6. Radial shrinkage of ZMT3 and NZC vs uniaxial pressure.

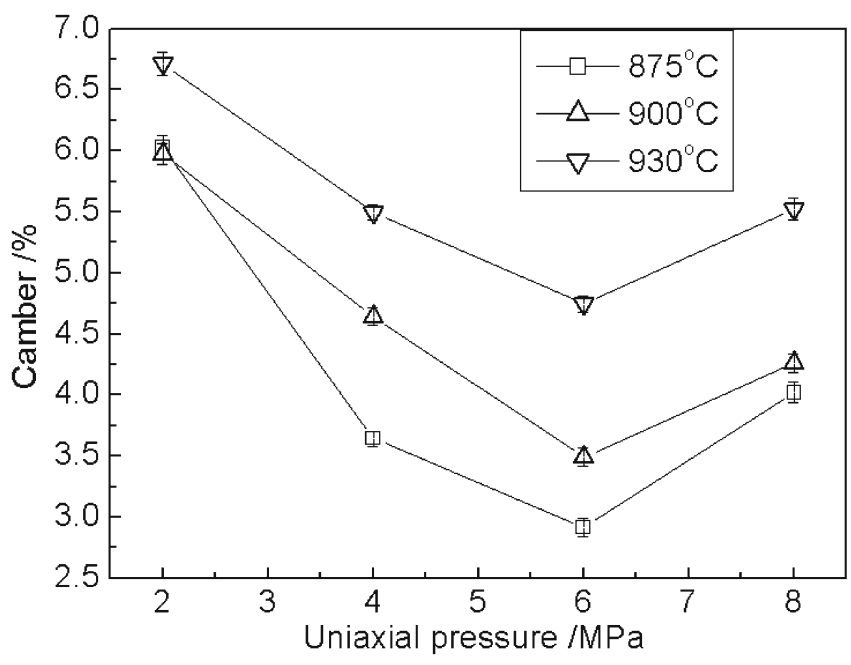

Figure 7. Camber development with uniaxial pressure and sintering temperature.

geometric averages of five samples). It can be seen that the shrinkage curvature shows an inverse quadratic dependence on the applied pressure. If the two materials are applied at different pressures at the same time, the shrinkage difference may be zero or be reduced. For example, if the uniaxial pressure bearing on ZMT3 is $4 \mathrm{MPa}$ and the uniaxial pressure bearing on NZC is $6 \mathrm{MPa}$ at the same time, the shrinkage difference will be zero. It is clear that the shrinkage of NZC is very close to that of ZMT3 when the pressure is at $2 \sim 6 \mathrm{MPa}$. In order to ensure the camber decrease, uniaxial pressure of 2 8 MPa was applied on layer composite because ZMT3 has higher radial shrinkage close to that of NZC in this range of pressure (figure 6).

Figure 7 shows camber development of ZMT3/NZC with uniaxial pressure and sintering temperature (The experimental values were geometric averages of five samples). Obviously, the higher the uniaxial pressure is, the smaller the camber is within uniaxial pressure range of $2 \sim 6 \mathrm{MPa}$. But 


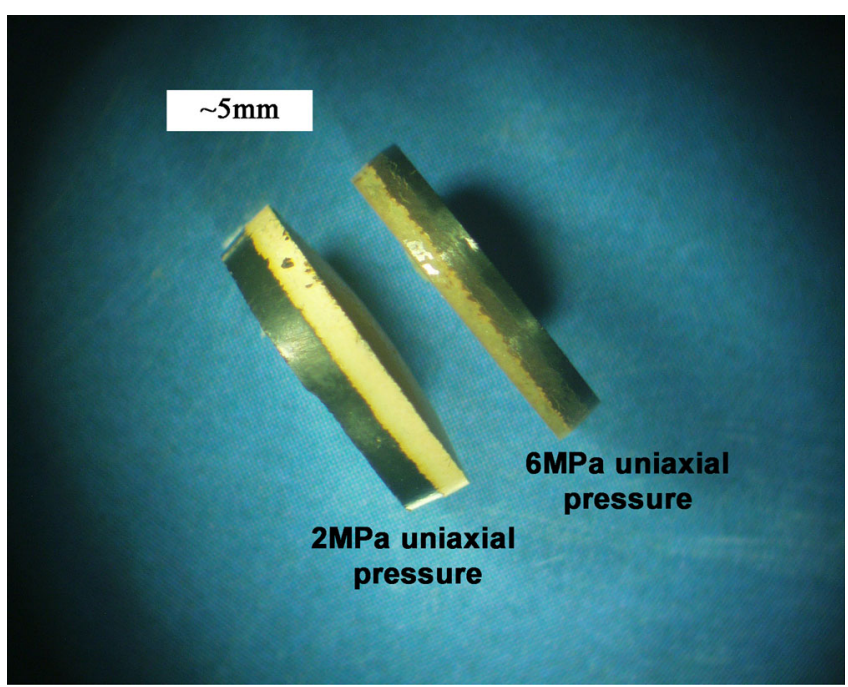

Figure 8. Photograph of ZMT3/NZC co-fired sample with different uniaxial pressures.

for the uniaxial pressure of $6 \sim 8 \mathrm{MPa}$, the camber becomes bigger. Generally, for compression moulding, when the pressure exceeds a critical value, too much pressure will lead to elastic deflection. When the pressure is removed, the body tends to restore to the original shape. In this work, NZC and ZMT3 bodies have different critical pressures. When the pressure is bigger than $6 \mathrm{MPa}$, NZC body shows strong elastic deformation and the shrinkage decreases, whereas ZMT3 body does not reach the critical value and the shrinkage continues to rise. As a result, the camber becomes bigger.

Figure 8 shows macrograph of ZMT3/NZC co-fired sample with different uniaxial pressures. It can be seen that the camber of ZMT3/NZC co-fired sample applied at $6 \mathrm{MPa}$ uniaxial pressure is decreased obviously compared with the sample applied at $2 \mathrm{MPa}$ pressure.

\section{Conclusions}

To summarize, investigation of sintering and camber development of the layer ZMT3/NZC composites demonstrate that the difference of shrinkage in the later stages of sintering is the key factor for camber deformation. In order to decrease the camber distortion, a new semi-fixed uniaxial pressure technique (SUP) was proposed. This technique uses uniaxial pressures by which different radial densities can be introduced when one layer's edge is clamped and the other is free while laminating two different layers. The results show that the SUP technique can decrease the camber obviously, and the camber curvature shows an inverse quadratic dependence on the applied pressure.

\section{Acknowledgments}

The work was supported by the National Natural Science Foundation of China (51072162 and 51372197), and the Scientific Research Program Funded by the Shaanxi Provincial Education Department (11JK0821).

\section{References}

Bordia R K, Zuo R, Guillon O, Salamone S M and Rodel J 2006 Acta Mater. 54111

Cai P Z, Green D J and Messing G L 1997a J. Am. Ceram. Soc. 80 1929

Cai P Z, Green D J and Messing G L 1997b J. Am. Ceram. Soc. 80 1940

Chang J, Guillon O, Rodel J and Kang S 2007 J. Eur. Ceram. Soc. 273127

Gao F, Yang Z P, Hou Y D and Tian C S 2003 J. Mater. Sci. 38 1523

Green D J, Guillon O and Rodel J 2008 J. Eur. Ceram. Soc. 281451

Hagymasi M, Roosen A, Karmazin R, Dernovsek O and Haas W 2005 J. Eur. Ceram. Soc. 252061

Jean J H and Lee C H 1999 Jpn J. Appl. Phys. 383508

Jiao X, Zhong C, Zhang S, Liu X and Li B 2010 J. Mater. Sci. 45 3331

Liu X C, Gao F, Zhao L and Tian C S 2007 J. Alloys Compd $\mathbf{4 3 6}$ 285

Liu X C, Gao F, Liu J and Tian C S 2009a J. Alloys Compd 470 269

Liu X C, Gao F and Tian C S 2009b J. Alloys Compd $\mathbf{4 8 6} 743$

Liu X C 2012 J. Electroceram. 2956

Merja T, Jari J and Heli J 2010 Compos. Struct. 93179

Seo S H and Oh J H 1999 IEEE Trans. Magn. 353412

Zhou H, Wang H, Li K and Yao X 2008 J. Mater. Sci. 433725 